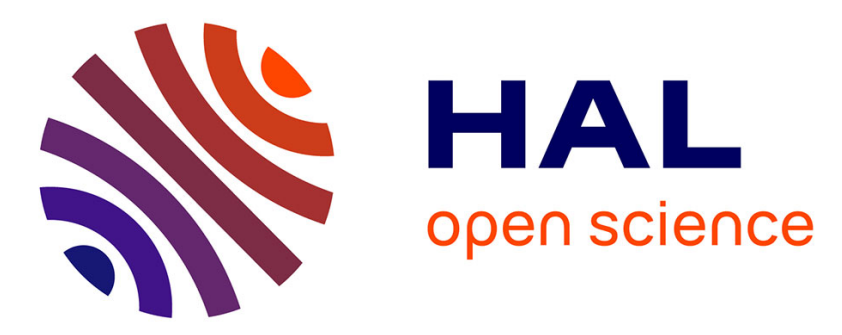

\title{
Etude diachronique du conditionnel passé ou l'origine de la contrefactualité
}

\author{
Adeline Patard, Natalia Grabar, Walter de Mulder
}

\section{To cite this version:}

Adeline Patard, Natalia Grabar, Walter de Mulder. Etude diachronique du conditionnel passé ou l'origine de la contrefactualité. Journal of French Language Studies, 2015, Les marqueurs de temps, aspect et modalité en diachronie, 25 (02), pp.189-211. 10.1017/S0959269515000101 . hal-01707894

\section{HAL Id: hal-01707894 \\ https://hal.science/hal-01707894}

Submitted on 13 Feb 2018

HAL is a multi-disciplinary open access archive for the deposit and dissemination of scientific research documents, whether they are published or not. The documents may come from teaching and research institutions in France or abroad, or from public or private research centers.
L'archive ouverte pluridisciplinaire HAL, est destinée au dépôt et à la diffusion de documents scientifiques de niveau recherche, publiés ou non, émanant des établissements d'enseignement et de recherche français ou étrangers, des laboratoires publics ou privés. 
Patard Adeline, Natalia Grabar, Walter De Mulder, 2015, «Etude diachronique du conditionnel passé ou l'origine de la contrefactualité », Journal of French Language Studies 25/2, 189-211.

\title{
Etude diachronique du conditionnel passé ou l'origine de la contrefactualité ${ }^{1}$
}

\author{
Adeline Patard \\ Natalia Grabar \\ Walter De Mulder
}

\section{Résumé}

L'article propose d'étudier diachroniquement le conditionnel passé (noté CONDPA) qui a pour particularité de pouvoir signifier contextuellement la contrefactualité (ex. Si j'avais su, je ne serais pas venu.). Le but de cette étude est de retracer, à travers une analyse de corpus, l'émergence de cette interprétation contrefactuelle qui s'est généralisée en français à partir du $17^{\text {ème }}$ siècle. Ce changement coïncide avec l'acquisition d'une valeur de passé aoriste, correspondant au dernier stade du chemin d'évolution des formes parfaites décrit par Bybee et al. (1994). L'émergence de la contrefactualité est analysée comme le résultat de la conventionnalisation d'inférences (cf. Heine 2002).

\section{Introduction}

Forme composée du conditionnel présent, le conditionnel passé (noté désormais CONDPA) n'a pris sa place actuelle dans le paradigme des temps verbaux que tardivement : il n'est pas attesté avant le $12^{\text {ème }}$ siècle et, selon Wagner $(1939: 231)$, ce n'est qu'au $18^{\text {ème }}$ siècle que son emploi s'est finalement imposé dans les constructions conditionnelles contrefactuelles [si plus-que-parfait, conditionnel passé]. L'étude de ce temps est généralement annexée à celle du conditionnel présent ${ }^{2}$. Dans les descriptions qui lui sont consacrées, le CONDPA est souvent présenté comme une simple version parfaite ('perfect') du conditionnel présent dont il ne se distingue que par les sens d'accompli et de passé qui caractérisent l'interprétation des formes composées.

Dans l'étude diachronique que nous proposons, nous tenterons de montrer que le CONDPA n'est pas uniquement un conditionnel parfait ou passé. Il se démarque des autres tiroirs verbaux par un sens spécifique ${ }^{3}$, contrefactuel ${ }^{4}$, qui s'est conventionnalisé à partir du $17^{\text {ème }}$ siècle et qui est devenu, comme nous le montrerons, l'interprétation prototypique de ce tiroir. On peut illustrer cette interprétation à l'aide de la phrase suivante :

(1) Si j'avais su, je ne serais pas venu.

\footnotetext{
${ }^{1}$ Nous tenons à remercier les relecteurs anonymes pour la pertinence de leurs commentaires qui ont largement contribué à améliorer la qualité de l'article. Les erreurs restantes sont les nôtres.

${ }^{2}$ L'étude de Wagner (1939) fait à cet égard figure d'exception : l'auteur consacre en effet plusieurs pages à la diachronie du CONDPA et à son usage dans les phrases hypothétiques.

${ }^{3}$ Nous utilisons ici le mot 'sens' dans une acception large permettant de désigner aussi bien l'invariant sémantique d'une forme que l'une de ses interprétations contextuelles. Le terme 'valeur' sera plus spécifiquement utilisée pour renvoyer à l'invariant sémantique, le terme 'interprétation' sera quant à lui réservé au sens contextuel attaché à des énoncés particuliers.

${ }^{4}$ Cette interprétation peut également être produite par l'usage de l'imparfait dans certains contextes (voir p. ex. Berthonneau et Kleiber, 2003 ; Bres, 2006 ou Patard et De Mulder, 2014).
} 
Le verbe au CONDPA serais venu renvoie à une situation contrefactuelle, autrement dit le CONDPA indique que non $p:$ le procès venir décrit un état de fait (passé) contraire à la réalité.

L'objectif de l'article est de rendre compte du développement de cet emploi contrefactuel du CONDPA à travers l'étude d'un corpus diachronique. L'article s'organise en trois parties. Dans la première partie, nous présentons le corpus et la méthodologie utilisée pour extraire et analyser les occurrences de CONDPA du corpus. Dans la seconde partie, nous résumons nos principales observations sur l'évolution de la valeur aspectuelle du CONDPA et l'émergence de son interprétation contrefactuelle. Dans la troisième et dernière partie, nous proposons une hypothèse expliquant l'émergence du sens contrefactuel en termes de conventionnalisation d'inférences.

\section{Corpus et méthodologie}

\subsection{Présentation du corpus}

Le corpus utilisé est un ensemble de textes que nous avons recueillis sous format électronique de façon à pouvoir procéder à des extractions ciblées de formes. Ce corpus s'étend du $11^{\text {ème }}$ au $20^{\text {ème }}$ siècle, compte environ 9,8 millions de mots et contient 152 textes répartis en 10 sous-corpus correspondant chacun à un siècle. Les dix sous-corpus ont des tailles variables qui sont fonction du nombre de textes que nous avons pu recueillir en format électronique ${ }^{5}$ (cf. figure 1). Du point de vue des genres représentés, le corpus n'est pas homogène mais présente quand même une relative continuité de siècle en siècle, ce qui permet de retracer les évolutions historiques d'une période à l'autre (cf. figure 2). En proportion, les genres littéraires sont bien représentés (récit, épopée, théâtre, poésie), viennent ensuite les genres narratifs 'non fictionnels' que sont les mémoires, les journaux et les récits historiques, suivis par des textes argumentatifs (essais et traités), les correspondances, et enfin une catégorie 'divers' qui inclut par exemple des textes de lois ou encore des textes inclassables comme Centuries de Nostradamus. Les figures 1 et 2 donnent la répartition du nombre de mots par siècle et la proportion en pourcentage par genre textuel.

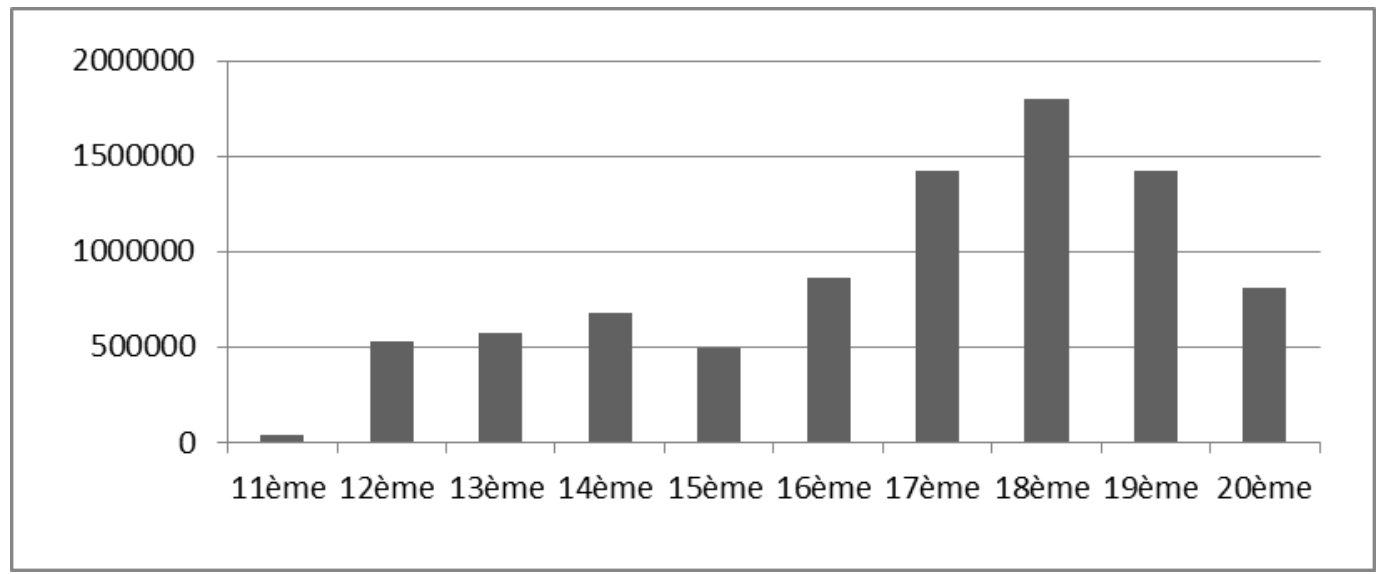

Figure 1. Nombre de mots par siècle

\footnotetext{
${ }^{5}$ On note ainsi que le sous-corpus du $11^{\text {ème }}$ siècle ne contient que 3 textes. Le corpus du $20^{\text {ème }}$ siècle est aussi beaucoup moins large que les deux sous-corpus précédents. La raison est la suivante : nous n'avons pu recueillir qu'un nombre limité de textes de la seconde moitié du $20^{\text {ème }}$ siècle car ces textes sont généralement encore sous droits et donc inaccessibles en format électronique. Nous avons néanmoins voulu conserver un certain équilibre avec les textes de la première moitié du siècle, aisément accessibles car libres de droits, en en limitant le nombre.
} 


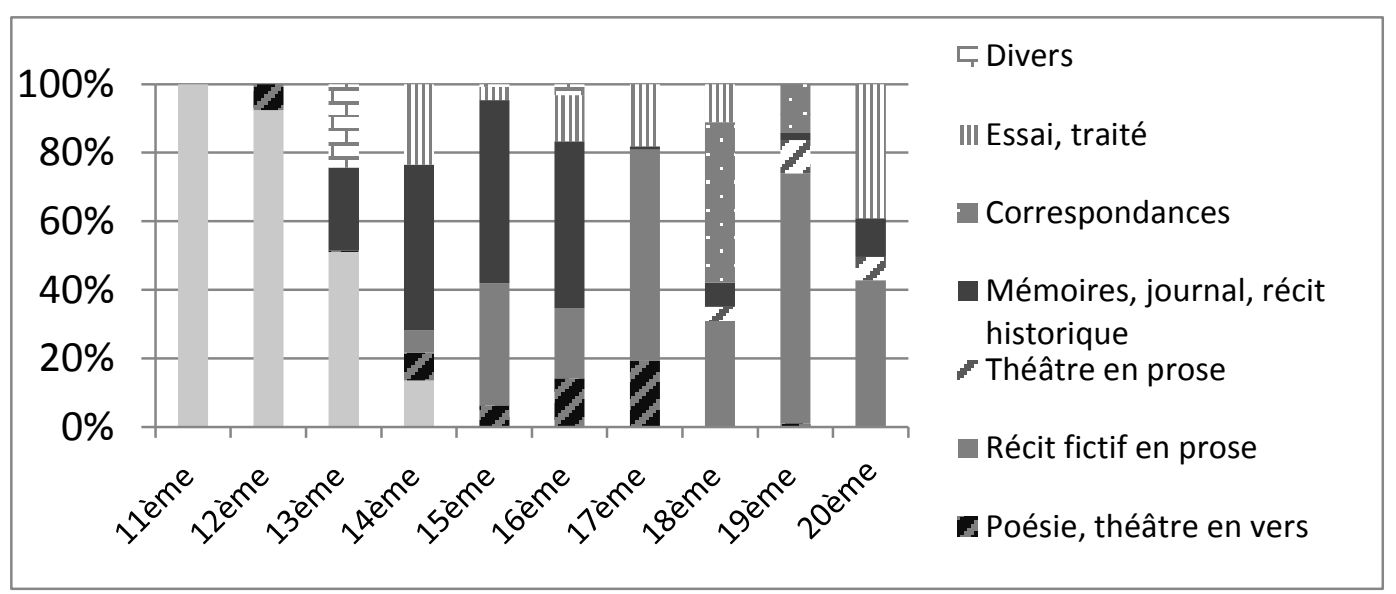

Figure 2. Proportion des genres textuels en pourcentage

\subsection{Extractions des conditionnels passés}

Pour extraire les CONDPA du corpus, nous avons adopté le protocole suivant :

(i) En nous fondant sur la grammaire de Buridant (2000), nous avons d'abord identifié les graphies des verbes avoir et être au conditionnel présentes dans le corpus : nous avons ainsi établi une liste de 90 graphies $^{6}$; cette étape vise à éviter au maximum les silences (occurrences pertinentes non repérées) dont le risque est plus élevé pour les périodes les plus anciennes dans la mesure où l'orthographe de la langue n'était pas encore fixée ;

(ii) nous avons ensuite relevé toutes les occurrences des graphies identifiées ainsi que leur cotexte (15 mots avant et après l'occurrence) ;

(iii) nous avons enfin trié manuellement les formes pour éliminer les bruits, c'est-à-dire les cas de CONDPR (sans participe passé) ou les cas de formes autres que des conditionnels.

Par ailleurs, nous avons utilisé la méthode de l'échantillonnage pour les quatre derniers siècles car les occurrences extraites se sont avérées trop nombreuses à traiter. Pour ce faire, nous avons sélectionné 100 occurrences de CONDPA par siècle à l'aide de suites aléatoires de façon à obtenir des échantillons représentatifs de chaque sous-corpus. Nous avons retenu le nombre de 100 occurrences pour les échantillons car les premiers sous-corpus non-échantillonnés comptaient entre 50 et 100 occurrences de CONDPA : de cette façon, nous avons pu obtenir un nombre comparable d'occurrences représentant chaque sous-corpus.

\subsection{Annotations des occurrences}

\footnotetext{
${ }^{6}$ Les 90 graphies identifiées sont : aroie, aroye, auroie, auroye, averoie, averoye, avraie, avreie, avroie, avroye, avroies, aroies, arois, aurais, auroies, aurois, averois, avreies, avroies, avroies, avrois, avereit, arait, aroit, aroyt, aurait, auroit, aurroit, auvrait, averoit, averroit, avrait, avreit, avroit, avrïens, aurions, averions, avrions, avrïons, arïez, ariés, ariez, auriez, auriez, averiez, avriez, avrïez, avriiez, avreient, aroient, aroyent, auraient, auroient, auroyent, avereient, averoient, avroient, serraie, esteroie, sereie, seroie, seroye, serreie, sereies, serais, seroies, serois, esteroit, sereit, serait, seroit, seroyt, serreit, serroit, serïens, serions, serïons, serïuns, esterïez, seriez, serïez, seriiez, sériés, estreient, serïent, seraient, sereient, seroient, seroyent, serreient.
} 
Pour déterminer l'importance de l'emploi contrefactuel du CONDPA à travers l'histoire du français, nous avons annoté les occurrences relevées en fonction du type d'emploi. Nous nous sommes inspirés de la typologie des usages du conditionnel proposé par Dendale (2001) pour distinguer cinq types d'emploi.

(i) L'emploi en discours indirect ou indirect libre (les emplois de 'futur du passé' de Dendale : ibid) :

(2) [...] le conseil d'Angleterre [...] luy [au roi]disoyent que, quant il auroit conquis [la Picardie], il pourroit bien essayer de conquerir Calaix et Guynes. (P. de Commynes, Mémoires, $15^{\text {s̀me }}$ siècle)

Le CONDPA opère un ancrage temporel relatif (et donc indirect) de la situation : celle-ci est à la fois ultérieure à une énonciation passée (ici exprimée par 'disoyent') et antérieure à un autre repère (exprimée par 'pourroit [...] essayer'). Le CONDPA correspond ainsi à la transposition dans le passé d'un futur antérieur.

(ii) L'emploi d'éventualité :

(3) Puis n'avroit garde de morir, ma dameisele, vostre amis, qui ceste herbe li avroit mis sor ses plaies et bien liee. (Ch. de Troyes, Perceval, $12^{\text {ème }}$ siècle)

'Votre amie, ma demoiselle, n'aurait plus à craindre de mourir, si on lui appliquait de cette herbe sur les plaies, en l'y attachant bien.'

La situation décrite est donnée comme hypothétique. Autrement dit, la proposition p n'est ni vraie ni fausse et l'on a, en adaptant la formule d'Haillet (2002: 46) ${ }^{7}$, 'ni p, ni non p'. Notons que la situation éventuelle se situe alors généralement dans le présent ou dans le futur. Néanmoins, ce n'est pas nécessairement le cas : elle peut aussi appartenir à l'époque passée lorsque le locuteur ignore ou n'est pas certain du cours des événements, comme dans l'exemple suivant (cf. 'et ne sçay si je l'ay dit ailleurs') :

(4) Je luy ose bien porter ceste louange (et ne sçay si je l'ay dit ailleurs, et quant je l'auroye dit, si vault-il bien de dire deux foiz) que jamais je ne congneü si saige homme en adversitéz. ( $\mathrm{Ph}$. de Commynes, Mémoires, $15^{\text {ème }}$ siècle)

'Je ne sais pas si je l'ai dit ailleurs, et quand je l'aurais déjà dit, il est préférable de le dire deux fois.'

(iii) L’emploi de contrefactualité :

(5) Si ma complaincte en vengeance estoit telle, [...] Croy qu'elle auroit desjà jecté fumée Du stile ardant, dont elle est alumée, Pour du tout rendre aussi noir que Charbon. (C. Marot, Elégies, $16^{\text {ème }}$ siècle)

'Si ma complainte était animée par la vengeance, $[\ldots]$ crois bien qu'elle aurait déjà jeté de la fumée du stylet ardant dont elle est allumée pour tout rendre aussi noir que charbon.'

La situation décrite est contraire à la réalité. On a donc, en adaptant la formule d'Haillet $(2002: 41)^{8}$, 'non p' : la proposition p n'est pas le cas. Cet emploi est, selon nous, propre au CONDPA. En effet, à la différence du CONDPA, le CONDPR ne permet jamais, en lui-même (p. ex. sans faire intervenir nos connaissances encyclopédiques ${ }^{9}$ ), d'exclure complétement la situation de la réalité.

\footnotetext{
${ }^{7}$ La formule originale d'Haillet est 'ni $\mathrm{A}$, ni non-A' où $\mathrm{A}$ correspond à une proposition décrivant un procès.

${ }^{8}$ Haillet appelle l'interprétation contrefactuelle 'l'effet de sens de type irréel'.

${ }^{9}$ Cf. Gosselin (1999 : 40).
} 
Remarquons enfin que, contrairement à l'emploi d'éventualité, l'emploi de contrefactualité est généralement associé à l'époque passée. Toutefois, la situation contrefactuelle peut parfois se localiser dans le présent ou le futur si le contexte linguistique le suggère (cf. encore dans l'exemple (6)).

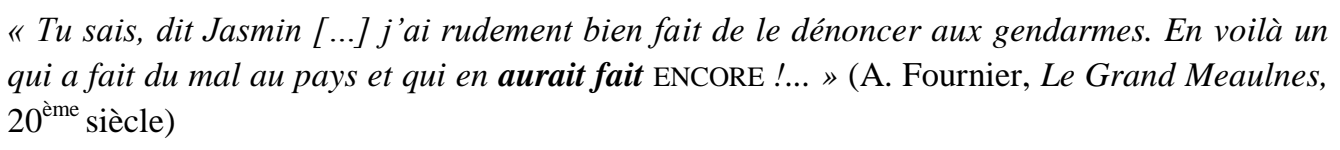
qui a fait du mal au pays et qui en aurait fait ENCORE !... » (A. Fournier, Le Grand Meaulnes, $20^{\text {ème }}$ siècle)

(iv) L'emploi d'atténuation : la forme conditionnelle permet d'atténuer un acte de langage (le plus souvent une requête) représentant une menace potentielle pour la face de l'interlocuteur. Nous n'avons trouvé aucune occurrence claire de cette catégorie d'usage même si, intuitivement, nous pensons que le CONDPA est aussi apte que le CONDPR à exprimer l'atténuation (ex. J'aurais voulu vous poser une question.).

(v) L'emploi d'évidentialité (ou de médiativité) :

Est-ce là, comme on le répète souvent, la continuation d'un mouvement de pensée qui débute chez Plotin? Plotin aurait intercalé, entre le premier principe et le monde, les hypostases de l'intelligence et de l'âme [...] ses successeurs, selon le même procédé, auraient intercalé d'autres termes comme on intercale des points [...]. (E. Bréhier, Histoire de la Philosophie, $20^{\text {ème }}$ siècle)

Ici, le CONDPA présente l'information véhiculée par l'énoncé comme étant empruntée à une source distincte du locuteur et donc comme non prise en charge par ce dernier. L'exemple cité comprend les deux uniques occurrences de notre corpus ${ }^{10}$.

Nous avons également voulu vérifier l'observation de Wagner (1939) selon laquelle la valeur aspectuelle parfaite du CONDPA s'est érodée au profit d'une valeur temporelle. Cette observation va dans le sens du chemin d'évolution des formes parfaites formulé comme suit par Bybee et al. (1994: 105) :

$$
\text { résultatif }^{(\mathrm{i})}>\text { antérieur }^{(\mathrm{ii})}>\text { passé }^{(\mathrm{iii})}
$$

Selon cette hypothèse, les formes parfaites qui sont fondées sur être ou avoir (i) sont originellement des constructions résultatives qui référent à des états résultants, (ii) puis elles acquièrent la capacité de renvoyer à une situation antérieure mais avec une pertinence actuelle ('current relevance'), (iii) elles expriment enfin, à un stade ultime, l'ancrage dans l'époque passée.

Pour vérifier cette hypothèse, nous avons annoté les occurrences relevées en fonction de leur interprétation d'antériorité et/ou de passé.

(i) L'interprétation d'antériorité. Le sens d'antérieur tel qu'il est entendu par Bybee et al. (1994), i.e. la pertinence actuelle d'une situation antérieure, est peu applicable à l'analyse de textes anciens : il est difficile en effet d'apprécier justement la pertinence actuelle de la situation décrite par le prédicat à partir de nos intuitions de locuteurs modernes. Aussi avons-nous adopté une définition plus stricte de l'antériorité qui présente l'avantage d'être aisément repérable, même dans les textes

\footnotetext{
${ }^{10}$ Néanmoins, des travaux antérieurs montrent que cet emploi remonte au $17^{\text {ème }}$ siècle (Bourova et Dendale, 2013) voire au $16^{\text {ème }}$ siècle (Kronning, 2014). Le peu d'occurrences observées s'explique par l'absence, dans notre corpus, du genre journalistique dans lequel le conditionnel évidentiel se rencontre fréquemment (d'où parfois l'appellation de 'conditionnel journalistique') et par la moindre proportion des textes argumentatifs (traités et essais) dans lesquels ce type d'emploi apparaît également.
} 
anciens, et qui constitue une manifestation possible du sens d'antérieur (c'est-à-dire parfait) tel qu'il est conçu dans Bybee et al. (1994). Nous avons ainsi codé pour l'interprétation d'antériorité les occurrences du CONDPA où le procès décrit précède un autre procès dénoté dans la même phrase. L'énoncé suivant en fournit un exemple typique :

(8) [...] s'il estoit couars, [li baillis] n'oseroit couroucier le riche homme qui avroit a fere contre le povre, ou il n'oseroit celui qui avroit mort deservie fere justicier. ( $\mathrm{Ph}$. de Beaumanoir, Coutumes de Beauvaisis, $13^{\text {ème }}$ siècle)

Le procès au CONDPA (avroit deservie), ici décrit dans une subordonnée, est donné comme antérieur au procès de la principale n'oseroit. Cet exemple illustre bien la valeur de pertinence ultérieure du procès accompli : l'acte antérieur de (mort) deservir est pertinent pour la situation ultérieure n'oser car il souligne la couardise du bailli qui refuse de rendre justice contre un homme puissant qui aurait commis un crime. En somme, lorsqu'un premier procès est posé comme antérieur à un second procès dans un énoncé, c'est que les conséquences du premier procès ont, d'une façon ou d'une autre, une pertinence pour le second procès.

Dans la discussion des résultats de ces annotations (cf. section 2.3), nous tiendrons compte de la stricte définition donnée à la valeur d'antériorité (uniquement une antériorité vis-à-vis d'un procès), ce qui signifie reconnaître, dans nos données, une sous-représentation des cas d'antérieurs tels qu'ils sont définis par Bybee et al. (1994).

(ii) L'interprétation de passé. Nous avons annoté comme telles toutes les occurrences qui renvoient à une situation révolue au moment de l'énonciation.

Si je n'avais pas été dans ce triste état, jamais Larsan ne se serait introduit dans la chambre de Mlle Stangerson ce soir-là (G. Leroux, Le mystère de la chambre jaune, $20^{\text {ème }}$ siècle)

Le choix de cette définition est également méthodologique puisqu'il facilite le traitement des occurrences en permettant de ne pas distinguer les interprétations purement passées des interprétations 'accomplies' avec un procès antérieur ayant une pertinence actuelle au moment de l'énonciation. Cela signifie que notre conception des passés est plus large que celle de Bybee et al. (1994) et donc, qu'il faudra admettre une surreprésentation des passés dans nos données (cf. section 2.3).

Les choix méthodologiques que nous venons de justifier pour les interprétations antérieures et passées ont une autre conséquence : une même occurrence peut à la fois être annotée pour l'interprétation d'antériorité (si elle décrit un procès antérieur à un autre procès) et pour l'interprétation de passé (si le procès est antérieur à l'énonciation). C'est le cas de (10) :

[...] car enfin cette Princesse ne m'a point refusé son affection opiniastrément durant un an, pour l'accorder à un homme qui ne la luy auroit pas demandée, et qui ne seroit pas amoureux d'elle. (M. de Scudéry, Artamène ou le Grand Cyrus, $17^{\mathrm{mèe}}$ siècle)

Une dernière conséquence de notre méthodologie est qu'une occurrence peut n'être annotée ni pour l'interprétation d'antériorité, ni pour l'interprétation passée. Ce type d'occurrence se rencontre plus fréquemment dans les premiers sous-corpus allant du $11^{\text {ème }}$ au $16^{\text {ème }}$ que dans les derniers sous-corpus ultérieurs au $16^{\text {ème }}$ siècle (cf. figure 6 en section 2.3). Il s'agit principalement de deux cas de figure. Dans le premier cas le plus fréquent, le CONDPA semble associé à une valeur d'accompli - le procès est présenté comme ayant abouti à son terme - mais sans pour autant signifier l'antériorité du procès par rapport à un autre procès. Le CONDPA revêt alors souvent une tonalité expressive que l'on peut qualifier de 'mirative' : il permet de souligner le caractère remarquable ou inattendu de 
l'accomplissement de la situation (ou de son non-accomplissement dans le cas d'énoncés négatifs) (cf. (11)) ; aujourd'hui, c'est le plus souvent une forme simple qui lui serait préférée :

(11) Bien me deüst on escorchier, je ne vos avroie hui retrait la honte que je li ai fait. (Anonyme, Le Roman de Renart, Branche I, $12^{\text {ème }}$ siècle)

'On aurait bien dû m'écorcher, je ne pourrais vous raconter en une journée [lit. je n'aurais pas raconté aujourd'hui] tous les outrages que je lui ai fait subir.'

Le second cas, moins fréquent, correspond aux interprétations résultatives du CONDPA que l'on rencontre notamment avec des adverbiaux renvoyant à un état résultant; ce type d'occurrence sera illustré et analysé en section 2.4.

Dans la section suivante nous présentons et discutons les principales observations permises par l'étude du corpus.

\section{Observations}

\subsection{Première attestation et fréquence}

Tout comme Wagner (1939), nous n'avons pas observé le CONDPA avant les textes du $12^{\text {ème }}$ siècle. L'attestation la plus ancienne du corpus est l'exemple déjà donné par Wagner du Couronnement de Louis :

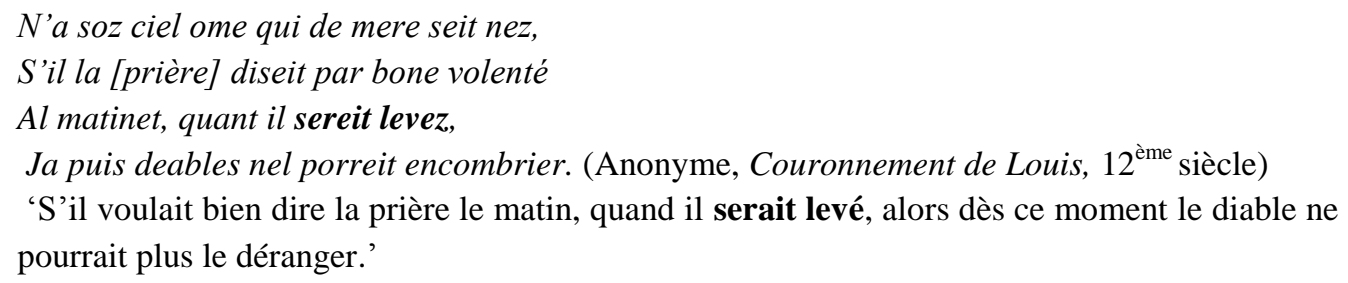

Cet exemple illustre bien le contexte d'emploi et l'interprétation typique du CONDPA en français médiéval. Le CONDPA apparaît dans une phrase hypothétique en si où la situation décrite est présentée comme hypothétique mais éventuellement réalisable (valeur d'éventualité) et donnée comme antérieure à une autre situation (valeur d'antériorité (vis-à-vis d'un autre procès)).

Concernant la fréquence du CONDPA à travers les différents sous-corpus, deux périodes se distinguent nettement (cf. figure 3):

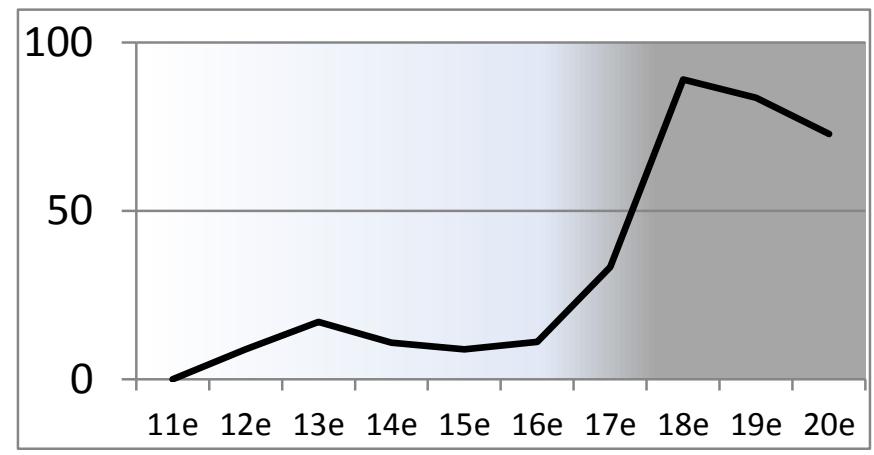

Figure 3. Fréquence (n occurrences par 100000 mots)

(i) jusqu'au $16^{\text {ème }}$ siècle, le CONDPA a une fréquence assez basse : autour de 10 occurrences par 100000 mots ; 
(ii) à partir du $17^{\text {z̀me }}$ siècle, le CONDPA voit sa fréquence augmenter fortement ; elle atteint plus de 70 occurrences par 100000 mots à partir du $18^{\text {ème }}$ siècle. Si l'analyse de Wagner (cf. section 1.3) est juste, cette augmentation de la fréquence doit être corrélée à l'érosion de la valeur aspectuelle du CONDPA. C'est ce qu'indiquent les observations suivantes.

\subsection{Types d'emploi}

L'évolution de la proportion des différents types d'emploi définis précédemment (cf. section 2.3) est représentée dans la figure $4^{11}$.

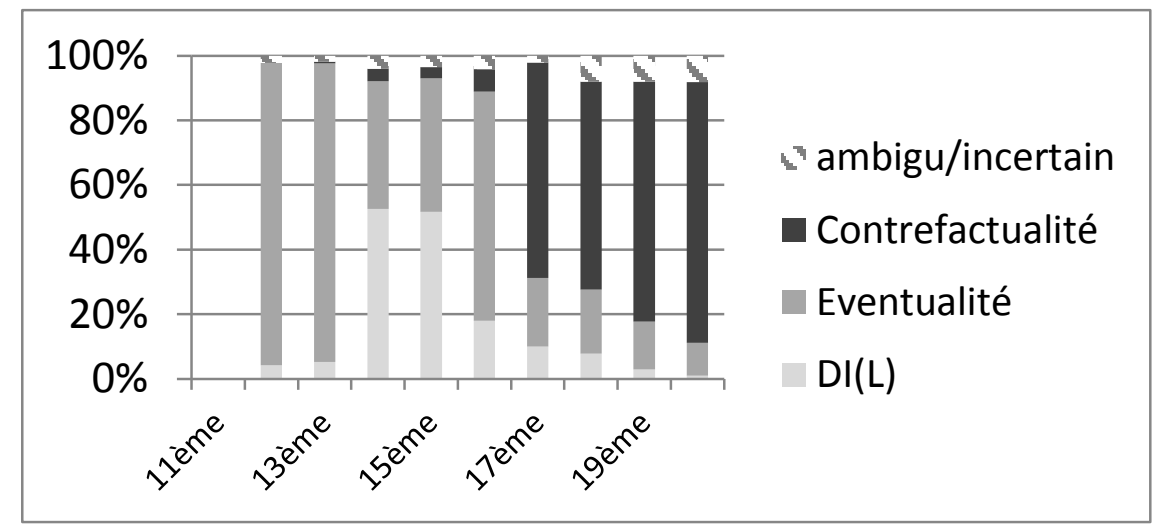

Figure 4. Evolution de la proportion des types d'emplois (en pourcentage)

Avant de commenter l'évolution des types d'emploi du CONDPA, une remarque s'impose : deux sous-corpus (ceux des $14^{\text {ème }}$ et $15^{\text {ème }}$ siècles) présentent un biais. En effet, dans ces sous-corpus, les emplois en discours indirect et indirect libre sont surreprésentés (respectivement $53 \%$ et $52 \%$ des emplois), à cause de deux textes qui font un usage massif du discours rapporté : les Chroniques de Froissart au $14^{\text {ème }}$ siècle et les Mémoires de Philippe de Commynes au $15^{\text {ème }}$ siècle. En dehors de ces deux textes, les emplois en discours indirect ou indirect libre sont en proportions plus modestes. Néanmoins, celles-ci restent élevées par rapport aux autres sous-corpus (respectivement $38 \%$ des emplois pour le $14^{\text {ème }}$ siècle et $43 \%$ des emplois pour le $15^{\text {ème }}$ siècle), sans que nous ne puissions expliquer ce fait.

Concernant les deux autres emplois largement représentés (l'emploi d'éventualité et l'emploi de contrefactualité), on note un basculement qui coïncide avec l'accroissement de fréquence précédemment observé : jusqu'au $16^{\text {ème }}$ siècle, les emplois d'éventualité (en gris) sont en majorité alors qu'à partir du $17^{\text {ème }}$ siècle, lorsque le CONDPA devient plus fréquent, ce sont les emplois de contrefactualité (en noir) qui constituent la majorité et les emplois d'éventualité deviennent une minorité. La figure 5, qui représente l'évolution de la fréquence relative de chaque type d'emploi, révèle un autre fait: si l'emploi d'éventualité décroît en proportion (cf. figure 4), sa fréquence ne diminue pas mais se maintient largement par la suite (jusqu'à 17,3 occurrences par 100000 mots au $18^{\text {ème }}$ siècle). Cette observation signifie que le sens d'éventualité n'a pas reculé en français moderne,

${ }^{11}$ La proportion des occurrences dont l'interprétation est incertaine ou ambivalente est également indiquée, en hachuré. 
seulement sa proportion est devenue largement minoritaire par rapport au sens de contrefactualité nouvellement développé.

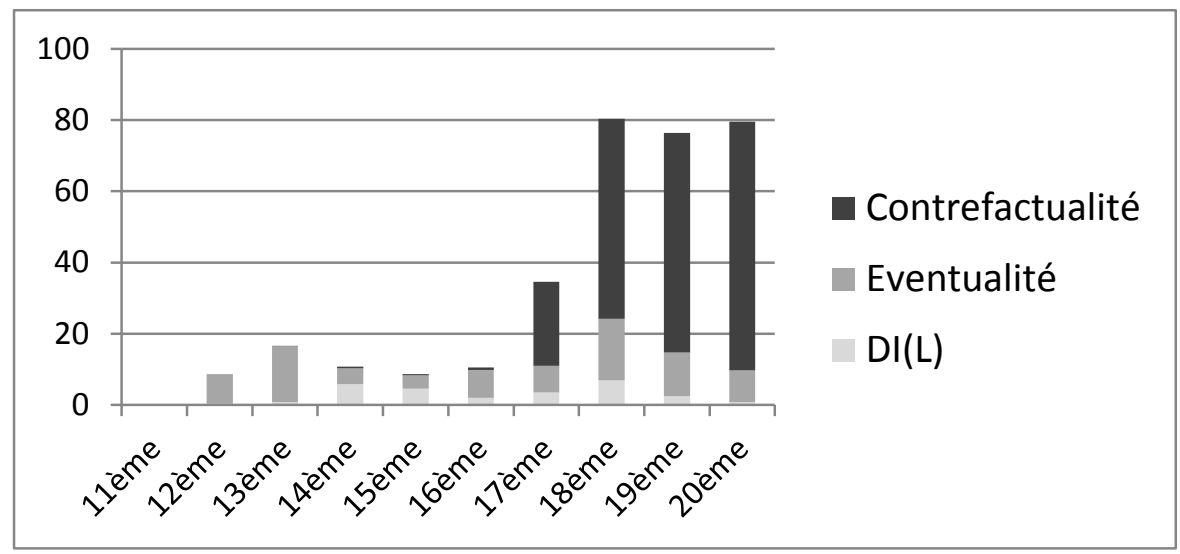

Figure 5. Evolution de la fréquence relative des types d'emplois (nombre d'occurrence par 100000 mots)

En conclusion, l'étude des types d'emplois montre que le CONDPA connaît à partir du $17^{\text {ème }}$ siècle une expansion qui se manifeste par l'emploi plus fréquent du CONDPA en contexte contrefactuel. Dans la section suivante, nous montrons que l'essor de cet emploi modal est lié au recul de l'interprétation aspectuelle du CONDPA au profit d'une interprétation temporelle.

\subsection{Interprétations aspectuelle et temporelle}

La figure 6 donne l'évolution de la proportion des interprétations antérieures et passées du CONDPA telles qu'elles ont été définies en section 2.3.

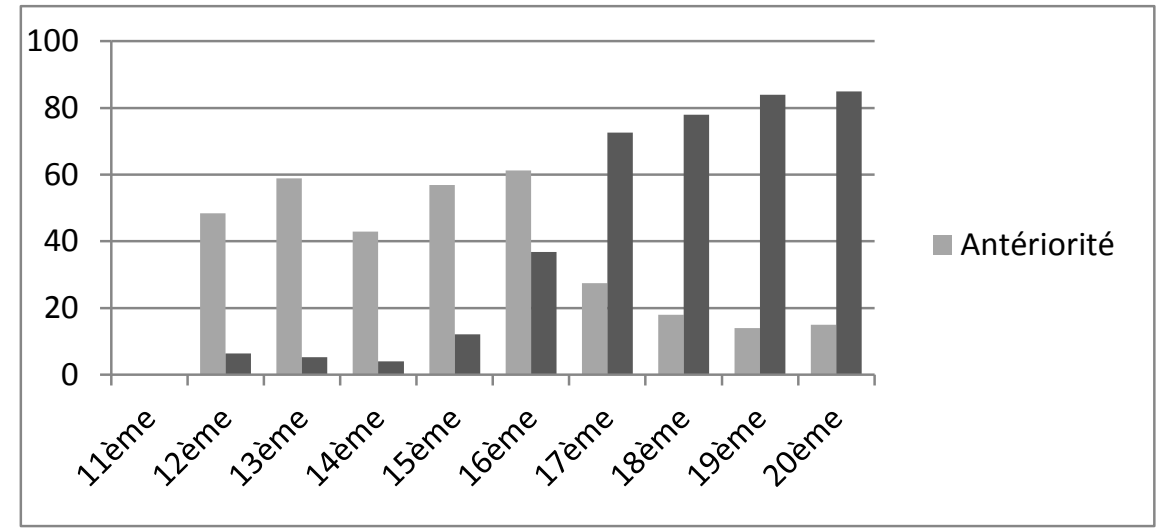

Figure 6. Evolution des interprétations antérieures et passées (en pourcentage)

Nous retrouvons dans ces données le basculement observé précédemment au $17^{\text {ème }}$ siècle. Jusqu'à ce siècle, le CONDPA s'applique le plus souvent à des procès qui sont donnés comme antérieurs à d'autres procès décrits dans le même énoncé. Comme nous l'avions proposé en section 1.3., l'antériorité vis-à-vis d'un autre procès peut être vue comme une manifestation possible de la valeur 
parfaite : dans ce type de contexte, les suites du procès sont en effet présentées comme pertinentes pour un procès ultérieur (cf. la valeur de 'pertinence actuelle' décrite par Bybee et al., 1994). Ces faits suggèrent que le CONDPA avait vraisemblablement une valeur parfaite jusqu' au $16^{\text {ème }}$ au moins.

A partir du $17^{\text {ème }}$ siècle, les interprétations passées deviennent la majorité, tandis que les interprétations antérieures décroissent. Ces observations s'accordent avec les analyses de Wagner (1939) et de Bybee et al. (1994) :

(i) $\quad \mathrm{du} 12^{\text {ème }}$ jusqu'au $17^{\text {ème }}$ siècle, le CONDPA semble se caractériser par un aspect parfait : il s'emploie typiquement pour signifier que le procès dénoté a des conséquences pertinentes pour un procès ultérieur (cf. (8) cité précédemment) ;

(ii) à partir du $17^{\text {ème }}$ siècle, le sens de parfait semble progressivement céder la place à un sens plus temporel ${ }^{12}$.

Autrement dit, le CONDPA est au stade 'parfait' au moins jusqu'au $17^{\text {ème }}$ siècle, période où le stade final de passé s'amorce avec la multiplication des contextes à ancrage passé.

Une question demeure néanmoins : le CONDPA a-t-il été à ses débuts une construction résultative comme le prévoit la théorie de Bybee et al. ?

\subsection{Un stade résultatif?}

Un certain nombre d'éléments indiquent qu'en ancien français, le CONDPA fonctionnait dans certains contextes comme une construction résultative.

Le premier indice est la co-occurrence du CONDPA avec des adverbiaux de temps renvoyant à des états résultants. Nous en avons dénombré deux types dans le corpus.

Le CONDPA peut d'abord se combiner avec des adverbiaux à orientation future qui dénotent l'atteinte de la borne initiale de l'état résultant. C'est notamment le cas de l'adverbe tost $^{13}$ qui est assez fréquemment employé avec le CONDPA pour indiquer que le début de l'état résultant est rapidement atteint. Par exemple, en (13), tost signale que le royaume serait rapidement plongé dans la misère, si le roi n'était craint des peuples dominés :

(13) Se Normanz nel cremeient, Engleis e Angevin E Bretun e Waleis, Escot e Peitevin, Mult avreient TOST fait tut le regne frarin. (Gu. de Pont-Sainte-Maxence, Vie de Saint Thomas Beckett, $12^{\text {ème }}$ siècle)

'Si le roi n'était craint de ses divers peuples, Normands, Anglais, Angevins, Bretons, Gallois, Ecossais et Poitevins, ils auraient BIENTÔT réduit tout le royaume à la misère.'

Ensuite, le CONDPA peut être associé à des adverbiaux qui spécifient la durée de l'état résultant. Par exemple, dans l'énoncé (14), toz tens '(pour) toujours' dénote la permanence de l'état résultant décrit par le procès metre an vilté, c'est-à-dire le mépris de Turnus pour Lavinia.

\footnotetext{
et se il est morz et veincuz et Turnus soit a ce venuz qu'il te doie a feme prendre, sel pooit savoir ne antendre que eüsses cestui amé, TOZ TENS t'avroit mes an vilté. (Enéas, $12^{\text {ème }}$ siècle)

'et s'il est mort et vaincu et qu'il arrive que Turnus doive te prendre pour épouse, si celui-ci pouvait apprendre ou comprendre que tu as aimé celui-là, il te tiendrait en mépris (lit. t'aurait mis dans le mépris) POUR TOUJOURS.'
}

12 Néanmoins, comme nous l'avions remarqué en section 1.3., l'association du CONDPA avec une interprétation passée ne signifie pas que celui-ci est lui-même porteur de la valeur passée. Mais on peut à bon droit penser que la multiplication des contextes passés puisse aboutir in fine à la réanalyse du CONDPA, dans certains contextes, comme forme du passé, c'est-à-dire comme dénotant sémantiquement l'ancrage passée de la situation.

${ }^{13}$ Nous avons aussi relevé : ja 'déjà', sempres 'aussitôt' et tantost 'aussitôt'. 
Ces types d'adverbiaux sont relativement fréquents au $12^{\text {ème }}$ siècle (présents dans $10 \%$ des cas) puis disparaissent entre le $13^{\text {ème }}$ et le $14^{\text {ème }}$ siècle. Ces données suggèrent qu'à ses débuts, le CONDPA était bien une forme résultative, comme le postule le chemin de grammaticalisation de Bybee et al. (1994). Mais le CONDPA semble être très rapidement devenu une forme parfaite avec une interprétation majoritairement antérieure.

Cette analyse est en partie confirmée par l'étude de la classe aspectuelle des prédicats au CONDPA (cf. figure 7).

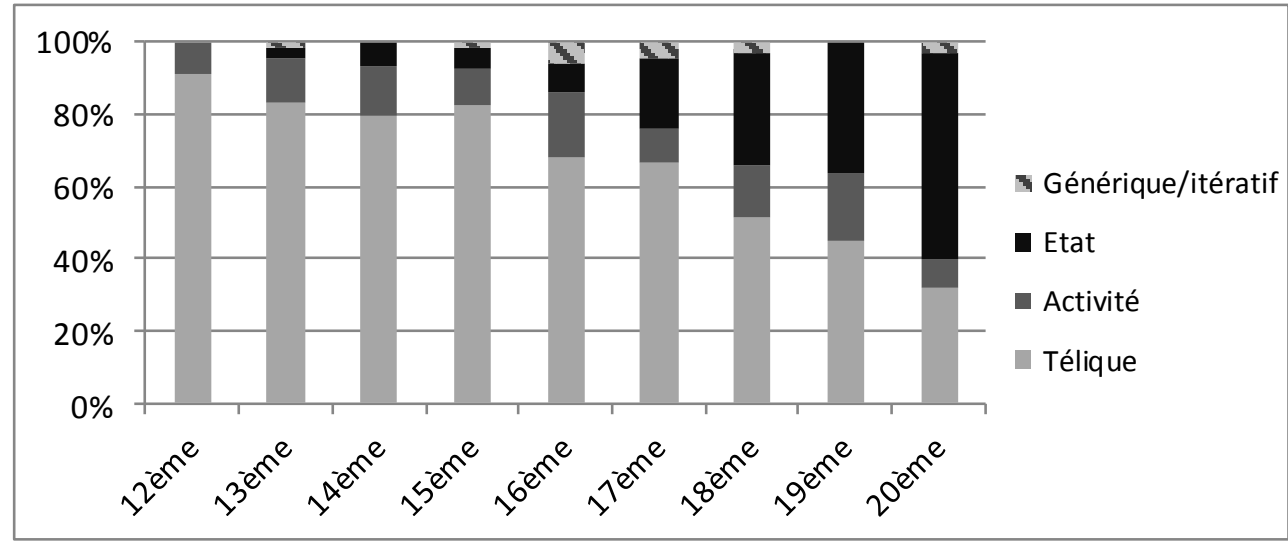

Figure 7. Les classes aspectuelles des prédicats (en pourcentage)

Le fait significatif concerne ici la proportion des prédicats statifs (en noir). Au $12^{\text {ème }}$ siècle, le CONDPA ne se combine jamais avec des procès de type «état ». Or, comme l'ont souligné différents auteurs (cf. entre autres Harris, 1978; Bybee et al., 1994 ou Carey, 1995), les constructions résultatives ne sont compatibles qu'avec les prédicats décrivant des actions débouchant sur un nouvel état et ne peuvent donc s'appliquer à des prédicats statifs qui n'engendrent pas de nouvel état. L'absence de procès statifs au $12^{\text {ème }}$ siècle abonde donc dans le sens d'un stade initial résultatif.

Le CONDPA s'est ensuite étendu aux prédicats statifs à partir du $13^{\text {ème }}$ siècle (cf. (ex. 15)), ce qui suggère que ce temps est progressivement devenu une forme parfaite à partir de cette époque :

(...) il ne demeure pas pour ce que li eritage ne puissent estre taillé en la maniere qu'il seroient taillié s'il estoient a un homme estrange qui onques n'avroit esté de leur commune. (Ph. De Beaumanoir, Coutumes de Beauvaisis, $13^{\text {ème }}$ siècle)

'Il n'est demeure pas moins que les propriétés peuvent être taxées de la manière qu'elles seraient taxées si elles appartenaient à un étranger qui n'aurait jamais été de la commune.' 
Puis, à partir du $17^{\text {ème }}$ siècle, la proportion des prédicats statifs s'est encore accrue jusqu'à représenter plus de $50 \%$ des prédicats au $20^{\text {ème }}$ siècle. Cela est notamment le fait de l'extension du CONDPA aux verbes modaux (ex. 15) dont on peut considérer qu'ils renvoient aussi à des états (nécessité, possibilité ou volonté) :

- Ne commencez pas à poser des questions, c'est une déplorable manie. Jacquemort aurait pu se fâcher, certes, mais son tempérament ne l'y poussa point. (B. Vian, L'Arrache-Cour, $20^{\text {ème }}$ siècle)

En effet, la modalité exprimée par le verbe modal s'apparente à une qualité homogène caractérisant la situation décrite (qui est vue comme étant nécessaire, possible ou voulue) et n'implique donc aucun changement ; les verbes modaux pourraient donc constituer une classe particulière de prédicats statifs (voir par exemple Mourelatos, 1978). Selon cette hypothèse, la multiplication des verbes modaux au CONDPA correspondrait au développement des interprétations passées et contrefactuelles de ce temps au détriment du sens parfait. En effet, le CONDPA ne renvoie plus à une situation donnée comme antérieure à une autre (réalisation contextuelle du sens parfait), mais à une situation n'ayant pas eu lieu dans le passé (contrefactualité passée). On remarque alors que c'est la situation qui est contrefactuelle, alors que la modalité décrite par le verbal modal est interprétée comme factuelle ${ }^{14}$. Pour reprendre l'exemple (15), la possibilité que Jacquemort se fâche a bien existé, mais c'est le fait qu'il se fâche qui ne s'est pas réalisé ('mais son tempérament ne l'y poussa point'). En somme, le sens de contrefactualité exprimée par le CONDPA ne porte pas sur la modalité, mais sur la situation dénotée par le verbe à l'infinitif.

En conclusion, l'étude de corpus semble corroborer pour le CONDPA le chemin de développement des parfaits (résultatif > antérieur (ou parfait) > passé) proposé par Bybee et al. (1994) :

(i) le stade résultatif est perceptible au $12^{\text {ème }}$ siècle avec l'impossibilité d'employer des prédicats statifs et la co-occurrence du CONDPA avec des adverbiaux référant à des états résultants ;

(ii) le stade antérieur se dessine à partir du $13^{\text {ème }}$ siècle avec l'extension du CONDPA à des prédicats statifs et une interprétation contextuelle majoritairement antérieure (sens parfait) ;

(iii) le stade ultime de passé s'ébauche au $16^{\text {ème }}-17^{\text {ème }}$ siècle avec des interprétations qui deviennent majoritairement passées et contrefactuelles et la multiplication des verbes modaux.

Dans la dernière partie, nous allons nous focaliser sur le passage du stade antérieur au stade de passé en tentant d'expliquer développement du sens contrefactuel qui caractérise aujourd'hui le CONDPA. Selon notre hypothèse, ce changement sémantique est le résultat de la conventionnalisation d'inférences pragmatiques.

\section{L'origine de la contrefactualité : analyse du changement linguistique}

\subsection{Modèle}

Notre hypothèse explicative s'inspire principalement du modèle inférentiel proposé par Heine (2002) pour rendre compte de l'évolution sémantique d'une forme grammaticale. Heine suggère un scénario en quatre étapes que l'on peut résumer (avec quelques adaptations) dans le schéma donné en figure 7.
Stade 1
Stade 2
Stade 3
Stade 4

\footnotetext{
${ }^{14}$ Néanmoins, avec des verbes désidératifs tel que vouloir, la modalité volitive peut aussi être interprétée comme contrefactuelle : ex. J'aurais bien voulu voir ça.
} 


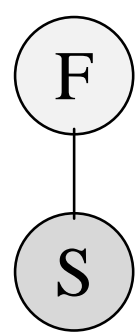

Situation initiale

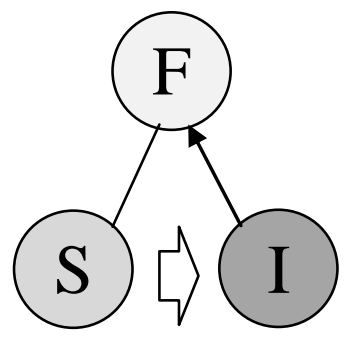

Inférence («bridging contexts »)

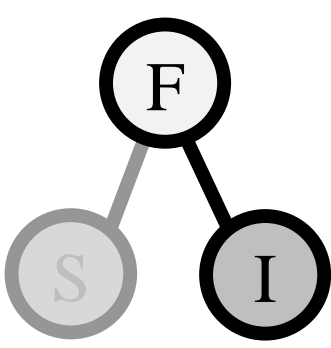

Conventionnalisation («switch contexts »)

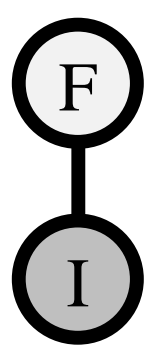

Changement sémantique achevé

Figure 8. Conventionnalisation d'inférence (adapté d'après Heine, 2002)

(i) Au stade 1, une unité linguistique associe une forme $\mathrm{F}$ à un sens $\mathrm{S}$ qui correspond à sa valeur sémantique.

(ii) Au stade 2, l'unité apparaît dans des contextes où le sens $\mathrm{S}$ donne lieu à une inférence I de telle sorte que la forme F y est également associée à l'interprétation I. Ces contextes sont appelés 'bridging contexts' par Heine.

(iii) Au stade 3, la forme F s'emploie de plus en plus pour exprimer l'inférence I. Avec la fréquence croissante de ces emplois (indiquée par le trait gras), la forme $\mathrm{F}$ est davantage associée à l'inférence I qui constitue, dans ces contextes, le sens que le locuteur entend communiquer. En conséquence, le sens source de l'unité linguistique passe à l'arrière-plan et l'interprétation I se conventionnalise. Ce stade correspond au stade des 'switch contexts' de Heine.

(iv) Enfin, au stade 4, lorsque l'inférence I est pleinement conventionnalisée, I est devenu le nouveau sens de la forme, c'est-à-dire sa valeur sémantique intrinsèque, le changement sémantique est alors achevé.

Ce scénario permet de rendre compte de l'évolution sémantique du CONDPA, comme nous le montrons dans la section suivante.

\subsection{De l'aspect au temps, de l'éventualité à la contrefactualité}

\subsubsection{Stade initial : éventualité et aspect parfait}

Pour retracer l'origine de la contrefactualité du CONDPA, nous prenons comme point de départ la situation qui se dessine à partir du $13^{\text {ème }}$ siècle. A ce stade, le CONDPA peut s'analyser compositionnellement comme une forme modale éventuelle (sens du morphème du conditionnel) et aspectuelle parfaite (sens de la construction composée ${ }^{15}$. Comme nous l'avons vu, le CONDPA s'emploie alors majoritairement pour exprimer l'antériorité de la situation par rapport à une autre

15 Nous faisons ici l'hypothèse, qui devra être développée et justifiée dans des travaux ultérieurs, que le CONDPA correspond en ancien français à deux constructions distinctes (au sens de Goldberg 2006) : la première correspond au discours rapporté où le CONDPA est associé à un sens de futur du passé accompli, la seconde est la construction hypothétique (avec ou sans protase) où le conditionnel est associé au sens éventuel puis au sens contrefactuel. Selon cette hypothèse, la morphologie du CONDPA est reliée dans la compétence des locuteurs à deux unités linguistiques différentes ayant chacune une valeur sémantique propre, soit temporelle soit modale. 
situation dénotée dans le contexte, par exemple en (17) l'antériorité d'emploier et recevoir par rapport à faire guerre :

Car donc avroie je chevalerie mauvesement emploie et receue de male hore, se je l'en fesoie guerre. (Anonyme, La Queste del saint Graal, $13^{\text {ème }}$ siècle)

'Si je m'opposais à lui, j'aurais mal employé et reçu pour mon malheur l'ordre de chevalerie.'

\subsubsection{Inférences passées et contrefactuelles}

Dès le $13^{\text {ème }}$ siècle, le CONDPA peut également s'employer dans des contextes passés et contrefactuels (des 'bridging contexts'), comme dans la phrase hypothétique suivante :

(18) Si lui dirent li pluiseurs que, se il eust esté au païs, que cil de l'Escorta n'auroient fait ce que il firent. (Anonyme, Chroniques de la Morée, $13^{\text {ème }}$ siècle)

'Ainsi la plupart lui dirent que s'il avait été dans le pays, les hommes de l'Escorta n'auraient pas fait ce qu'ils firent.'

Ici, la contrefactualité est marquée par l'usage du subjonctif plus-que-parfait ('eust esté') dans la protase et par le contexte droit 'n'auroient fait ce que il firent'.

Dans ce type d'exemple, le CONDPA semble aussi contribuer à cette interprétation grâce à son aspect parfait. Cette interprétation est le résultat de deux inférences successives : une implication sémantique passée (non annulable) puis une implicature conversationnelle contrefactuelle (annulable). D'abord, en présentant la situation dénotée comme antérieure à une autre situation passée (exprimée par 'dirent'), le CONDPA implique sémantiquement que celle-ci est elle-même passée: si une situation est antérieure à un moment passé, elle est elle-même nécessairement passée. Cette implication provient directement du sens encodé par l'énoncé, en l'occurrence le sens passé dénoté par le passé simple dirent et l'interprétation d'antériorité qui élabore contextuellement le sens parfait du CONDPA. Pour cette raison, cette implication passée ne peut être annulée (par le contexte droit par exemple).

Ensuite, l'interprétation passée jointe au sens d'éventualité entraîne une implicature contrefactuelle liée à notre expérience pratique du temps. En effet, depuis l'antiquité ${ }^{16}$, intellectuels et penseurs observent que l'homme conçoit le temps vécu comme un 'flux irréversible' sur lequel le présent subjectif opère une 'coupe modale' (Gosselin, 2009: 138) : le passé, irrévocable, s'oppose ainsi à l'avenir possible et indéterminé. Cette conception asymétrique du temps trouve comme parallèle l'idée que le passé est généralement connu (du moins connaissable) tandis que le futur nous est normalement inconnu (sauf dans le cas des situations prédictibles: événements cycliques, prophétisés etc.). En conséquence, lorsque qu'un locuteur évoque une situation passée et éventuelle (alors que le passé est généralement connu), il suggère par défaut que cette situation n'a pas été le cas, que celle-ci est contrefactuelle. C'est ce qu'illustre l'énoncé suivant où les conditionnels passés 'aurait rendu' et 'aurait fait' dénotent l'éventualité passée des situations décrites :

Pierre aurait rendu visite à Marie, cela lui aurait fait plaisir.

$\rightarrow$ Implicature contrefactuelle ('non p') : 'Marie n'a pas eu le plaisir d'avoir la visite de Pierre'.

Cette implicature, qui est fondée sur notre expérience pratique du temps, peut être annulée contextuellement si le locuteur signale qu'il ignore ce qui s'est passé (avec p. ex. 'mais, en vérité, je ne sais pas s'il a rendu visite à Marie') :

\footnotetext{
${ }^{16}$ Cf. Platon (Protagoras, 324b), ou encore Aristote (Ethique à Nicomaque, VII).
} 
$\rightarrow$ Interprétation éventuelle ('ni p, ni non p') : 'Marie a peut-être eu le plaisir d'avoir la visite de Pierre'.

Par conséquent, grâce à son fonctionnement pragmatique, le CONDPA est tout à fait approprié pour contribuer à l'interprétation contrefactuelle dans les contextes hypothétiques passés.

\subsubsection{Conventionnalisation des inférences}

A partir du $17^{\text {ème }}$ siècle, le conditionnel est de plus en plus associé à l'interprétation d'une contrefactualité passée qui est devenue majoritaire (cf. figure 4). Cette inférence permise par le CONDPA semble alors prendre le pas sur son sens source (éventualité et aspect parfait) : il s'agit du stade des 'switch contexts' décrit par Heine (2002). La mise à l'arrière-plan du sens source du CONDPA se traduit notamment par l'effacement de la valeur aspectuelle dans l'interprétation. En effet, comme nous l'avons vu (cf. figure 5), l'interprétation d'une antériorité, qui est une réalisation particulière du sens parfait, régresse de façon importante à cette époque. C'est ce qu'illustre l'exemple suivant :

Il tua tout ce qui s'opposa à son passage; et blessa Aribée en tant de lieux, qu'enfin il se seroit sans doute resolu de se rendre; si tout d'un coup une maison enflamée ne fust tombée si prés du lieu où ils combatoient, qu'Aribée en fut ensevelis. (M. de Scudéry, Artamène ou le Grand Cyrus, $17^{\text {ème }}$ siècle)

Ici l'événement décrit à l'aide du CONDPA ('se seroit resolu') n'est antérieur à aucun des autres événements dénotés dans les propositions adjacentes. Il s'inscrit au contraire dans une relation de narration avec le verbe de la proposition principale dont la subordonnée où il apparaît dépend : [blessa $>$ se serait resolu]. Ce recul des interprétations antérieures est le signe d'une mise à l'arrière-plan progressive de l'aspect parfait au profit du sens passé.

Concernant la valeur modale d'éventualité, elle est dès le départ supplantée (au stade II de l'inférence) par l'interprétation contrefactuelle qui vient préciser le statut épistémique de la situation : alors que la valeur d'éventualité permet au locuteur de ne pas se prononcer sur la réalité de $\mathrm{p}$ ('ni $\mathrm{p}$, ni non p'), l'interprétation contrefactuelle assigne une valeur de non-réalité à p ('non p'). Par conséquent, les deux sens ne peuvent pas être contigus dans l'interprétation : on a soit l'un soit l'autre. Cela signifie que, dès que le CONDPA donne lieu à une interprétation contrefactuelle, celle-ci 'déloge' et remplace la valeur éventuelle initiale.

A ce stade, les seuls éléments de sens qui demeurent au premier plan de l'interprétation sont donc les inférences permises par le CONDPA, c'est-à-dire l'implication passée et l'implicature contrefactuelle. Selon notre hypothèse, celles-ci se conventionnalisent à travers l'usage répété des contextes inférentiels de telle sorte que, dans la compétence des locuteurs, le CONDPA est progressivement associé à la contrefactualité passée (plutôt qu'à l'aspect parfait et à l'éventualité).

\subsubsection{Changement sémantique achevé?}

On peut ensuite se demander si la mutation sémantique du CONDPA est arrivée à son terme. Cela semble être le cas avec les verbes modaux au $20^{\text {ème }}$ siècle. En effet, en français moderne, les verbes modaux au CONDPA renvoient presque toujours à des situations contrefactuelles et passées sans qu'aucun autre marqueur temporel ou modal ne soit nécessaire pour obtenir cette interprétation, comme dans l'exemple suivant : 
Oh! non, je ne suis pas prêt... J'aurais dî dire au docteur Delbende que l'Église n'est pas seulement ce qu'il imagine, une espèce d'État souverain avec ses lois, ses fonctionnaires, ses armées (...) (G. Bernanos, Journal d'un curé de campagne, $20^{\text {ème }}$ siècle)

Dans cet énoncé, le CONDPA côtoie des présents de l'indicatif (suis, est, imagine) et aucun marqueur épistémique ne précise le statut modal de la situation décrite. Néanmoins, le procès dire au docteur Delbende est interprété comme n'ayant pas eu lieu dans le passé. Dans ce type de contexte, le CONDPA semble ainsi fonctionner comme un véritable marqueur de la contrefactualité passée.

On peut enfin noter que, dans quelques contextes, le CONDPA ne renvoie plus au passé, mais exprime uniquement la contrefactualité :

(23) Je vous aurais volontiers gardé à déjeuner... Mais je crains d'abuser de vos instants... (B. Vian, L'Arrache-cœur, $20^{\text {ème }}$ siècle)

Ici, le CONDPA aurais gardé décrit une situation contrefactuelle mais qui appartient à l'époque future, l'interprétation passée devient donc optionnelle. Il pourrait s'agir des prémisses d'une érosion sémantique du CONDPA vers une valeur uniquement contrefactuelle ${ }^{17}$.

\subsection{Dimension systémique du changement}

La question qui se pose maintenant est celle de la chronologie. Comme on l'a vu, le conditionnel passé manifeste, dès le $13^{\text {ème }}$ siècle, la possibilité d'exprimer pragmatiquement la contrefactualité passée. Pourtant, les interprétations contrefactuelles ne se sont multipliées qu'au $17^{\text {ème }}$ siècle (cf. la section 3.2.3 ci-dessous). Pourquoi ce délai ? Pour répondre à cette question, il faut prendre en compte la dimension systémique du changement sémantique en question. En ancien français, la contrefactualité passée (notamment dans les phrases hypothétiques) était exprimée par le subjonctif imparfait (24), même si ce dernier pouvait aussi signifier l'éventualité (25); autrement dit, le subjonctif imparfait était modalement ambigu puisqu'il pouvait renvoyer à une situation qui, en fonction du contexte, pourrait se réaliser ('ni p ni non p') ou bien aurait pu se réaliser ('non p') ${ }^{18}$ :

(24) S'altre·le desist, ja semblast grant mençunge. (Anonyme, La Chanson de Roland, $11^{\mathrm{ème}}$ siècle)

'Si un autre l'avait dit (lit. dît), cela semblerait (lit. semblât) un grand mensonge.'

Se tei ploüst, ici ne volisse estra. (Anonyme, La vie de saint Alexis, $11^{\mathrm{ème}}$ siècle).

'Si tu le voulais (lit. voulusses) bien, je serais (lit. fût) ailleurs qu'ici.'

Probablement à cause de cette ambiguïté sémantique, et dans le mouvement de développement des formes composées, le subjonctif plus-que-parfait commence, au $13^{\text {ème }}$ siècle, à remplacer le subjonctif imparfait dans ses emplois contrefactuels (26). Selon Wagner (1939: 226), cet emploi du subjonctif plus-que-parfait devient fréquent au $14^{\text {ème }}$ et $15^{\text {ème }}$ siècles et d'usage courant au $16^{\text {ème }}$ et au début du $17^{\text {ème }}$ siècle.

(26) Vos l'eüssiez destruit, se vos eüst pleü. (Anonyme, Aye d'Avignon, fin $12^{\text {ème }}$ - début $13^{\text {ème }}$ siècle)

'Vous l'auriez détruit (lit. eussiez détruit), si cela vous avait plu (lit. eût plu).'

Ainsi, à partir du $13^{\text {ème }}$ siècle, les locuteurs pouvaient choisir entre trois formes capables d'exprimer la contrefactualité passée: l'ancienne forme déclinante issue du latin: l'imparfait du subjonctif

\footnotetext{
${ }^{17}$ Néanmoins, comme le suggère Dahl (1997: 109) cité par Kronning (2009: note 18), l'effacement du sens passé pourrait aussi conduire à une réinterprétation éventuelle du sens contrefactuel.

${ }^{18}$ Cette ambiguité modale découle semble-t-il de la valeur temporelle indéfinie de l'imparfait du subjonctif qui pouvait indistinctement renvoyer au passé ou au présent (voir par exemple Wagner (1939: 295) ou Yvon (1958: 166-167)).
} 
(amassem > aimasse), et deux formes composées créées en français : le subjonctif plus-que-parfait (eusse aimé) et le conditionnel passé (aurais aimé). Or, comme on l'a vu, c'est le subjonctif-plus-queparfait qui conquiert dans un premier temps le terrain laissé par l'imparfait du subjonctif. Pour l'expliquer, on peut avancer une hypothèse qui prend en compte deux changements systémiques à l'œuvre au cours de cette période.

On peut d'abord rappeler que les formes composées de l'indicatif sont dans l'ensemble bien implantées dès le $11^{\text {ème }}$ siècle, où elles se rencontrent assez fréquemment dans les textes; cela est notamment vrai du passé composé, qui est devenu l'un des temps récurrents, à la fois dans le discours et dans le récit (voir par exemple Buridant, 1993 et 2000 ou Vetters, 2010). Cette forte fréquence des formes composées a certainement contribué au développement du subjonctif plus-que-parfait dans les hypothétiques contrefactuelles. Par comparaison, le conditionnel est loin d'être employé systématiquement en ancien français où il est encore fortement concurrencé par le subjonctif imparfait (cf. Patard et De Mulder, 2012: 30). C'est donc sans surprise que l'on voit, à partir du $13^{\text {ème }}$ siècle, le subjonctif imparfait être remplacé par le subjonctif plus-que-parfait (plutôt que par le CONDPA) $)^{19}$.

Quelques siècles plus tard, au $17^{\text {ème }}$ siècle, le CONDPR est d'un usage beaucoup plus systématique: les phrases hypothétiques en [si IMP, COND] ont en effet presque totalement évincé celles au subjonctif imparfait (Wagner, 1939: 344). Cela signifie qu'une asymétrie s'est peu à peu créée dans le paradigme des phrases hypothétiques: les hypothétiques éventuelles demandent maintenant des formes indicatives (l'imparfait et le conditionnel) et les contrefactuelles reposent sur l'emploi du subjonctif plus-que-parfait. On peut penser que cette asymétrie de mode s'est résolue par l'alignement analogique des phrases contrefactuelles sur le modèle des phrases éventuelles. Cela pourrait expliquer pourquoi le plus-que-parfait de l'indicatif et le CONDPA ont ensuite remplacé le plus-que-parfait du subjonctif à partir du $17^{\text {ème }}$ siècle dans les contextes contrefactuels.

Cette attraction a probablement été amplifiée par une autre évolution à l'œuvre à la même époque : le développement de l'interprétation aoristique des formes composées et notamment du passé composé (cf. p. ex. Caudal et Vetters, 2007 ou Vetters, 2010). Ce mouvement vers l'aoriste a sans doute favorisé, par analogie, l'interprétation passée du CONDPA ainsi que l'implicature contrefactuelle à laquelle elle donne lieu par défaut (cf. section 3.2). Suivant cette hypothèse, le CONDPA aurait plus facilement impliqué la contrefactualité pour un locuteur du $17^{\text {ème }}$ siècle que pour un locuteur du $13^{\text {ème }}$ siècle, d'où peut-être l'extension de son usage contrefactuel à l'époque classique.

En somme, ce n'est que dans un second temps que le CONDPA s'est étendu aux contextes contrefactuels au $17^{\mathrm{ème}}$ siècle, après une série de rééquilibrages touchant au système des temps et des modes du français.

\subsection{Bilan}

La théorie de la conventionnalisation d'inférences apporte une explication élégante à la mutation sémantique qu'opère le CONDPA autour du $17^{\text {zeme }}$ siècle. Cette période cruciale correspond au stade des 'switch contexts' de Heine (2002), où le CONDPA autorise des inférences passées et

\footnotetext{
${ }^{19}$ On peut penser que la pression analogique et le besoin de symétrie (une forme simple et une forme composée pour chaque temps) a pu également jouer un rôle dans le développement du subjonctif plus-que-parfait.
} 
contrefactuelles. C'est la haute fréquence de ces contextes de passage ${ }^{20}$ (cf. figures 4 et 5 ) qui entraîne la mise à l'arrière-plan du sens éventuel et parfait du CONDPA et la conventionnalisation du sens inférentiel passé et contrefactuel.

L'étude permet par ailleurs de préciser le modèle de Heine (2002). La diachronie du CONDPA nous apprend qu'il n'y a aucune différence qualitative entre les 'bridging contexts' et les 'switch contexts', mais que la différence est quantitative : les premiers sont relativement marginaux (du $13^{\text {ème }}$ au $16^{\text {ème }}$ siècle), tandis que les seconds sont plus fréquents et deviennent majoritaires (à partir du $17^{\text {ème }}$ siècle). Notre étude confirme donc également, si besoin était, l'importance du rôle de la fréquence dans le changement linguistique (cf. Bybee, 2006).

Nous avons enfin évoqué les raisons systémiques de cette extension qui mériteraient d'être plus approfondies : il s'agit d'une part du développement des formes parfaites et de leur transformation progressive en formes aoristiques, et, d'autre part, du retrait du mode subjonctif au profit du mode indicatif dans les phrases hypothétiques. La mutation sémantique du CONDPA apparaît donc comme une micro-évolution qui s'inscrit dans le cadre des changements macrostructurels plus larges touchant aux paradigmes des modes et des temps du français.

\section{En guise de conclusion}

L'étude proposée révèle que le CONDPA s'est intégré de façon singulière dans le paradigme des formes verbales du français. S'il a commencé son parcours comme le pendant 'parfait' du conditionnel présent, il est devenu à partir du $17^{\text {ème }}$ siècle une forme qui exprime très majoritairement la contrefactualité passée. Le CONDPA se présente donc en français moderne comme un marqueur à la fois temporel et modal et dont le sens contrefactuel n'est dénoté par aucune autre forme verbale.

Ce fait interroge, nous semble-t-il, son statut de temps verbal, d'autant plus que son interprétation passée peut être optionnelle (cf. (22)). Sans vouloir rouvrir le vieux débat sur le statut des formes conditionnelles (temps ou mode?, voir p. ex. Yvon 1952 ou Bondy 1958), notre travail souligne comme nombre de travaux précédents que les temps verbaux sont fréquemment associés à des valeurs modales et que cette dimension est souvent ignorée lorsque l'on décrit le paradigme des temps verbaux (cf. par ex. Gosselin, 2005 ; Barceló et Bres, 2006 ; Patard, 2007). La question devient épineuse avec une forme comme le CONDPA, dont le fonctionnement sémantique est fortement modal et ne peut donc se réduire à des traits temporels et aspectuels. En somme comment articuler temporalité et modalité dans le paradigme des temps verbaux ? Que faire des temps verbaux qui sont, en termes de fréquence, plus modaux que temporels? Autant de questions qui, nous l'espérons, inspireront des travaux futurs.

\section{Bibliographie}

Barceló, G. J. et Bres, J. (2006). Les temps de l'indicatif en français. Paris: Ophrys.

Berthonneau, A.-M. et Kleiber, G. (2003). Un imparfait de plus ... et le train déraillait. Cahiers Chronos, 11: 1-24.

Bondy, L. (1958). En marge des discussions sur les modes et les temps, Le Français Moderne, 26: 93100.

Bourova, V. et Dendale, P. (2013). Serait-ce un conditionnel de conjecture? Datation, évolution et mise en relation des deux conditionnels à valeur évidentielle. Cahiers Chronos 26: 183-200.

\footnotetext{
${ }^{20}$ Terme employé par Christiane Marchello-Nizia (2006) pour référer aux ‘switch contexts’ de Heine (2002).
} 
Bres, J. (2006). 'Encore un peu, et l'imparfait était un mode...' L'imparfait et la valeur modale de contrefactualité. Cahiers de praxématique, 47: 149-176.

Buridant, Cl. (1993). L'emploi des temps dans la chanson de geste "Ami et Amile". In: Actas do XIX Congreso Internacional de Lingüística e Filoloxía Románicas. La Coruña: Fundación Pedro Barrié de la Maza, pp. 645-698.

Buridant, C. (2000). Grammaire nouvelle de l'ancien français. Paris: Sedes.

Bybee, J., Perkins, R. et Pagliuca, W. (1994). The Evolution of Grammar. Tense, Aspect, and Modality in the Languages of the World. Chicago and Londres: The University of Chicago Press.

Bybee, J. (2006). From Usage to Grammar: The Mind's Response to Repetition. Language, 82.4: 711733.

Carey, K. (1995). Subjectification and the Development of the English Perfect. In: D. Stein et S. Wright (eds), Subjectivity and Subjectivisation. Linguistic Perspectives. Cambridge: Cambridge University Press, pp. 83-102.

Caudal, P. and Vetters, C. (2007). Passé composé et passé simple: sémantique diachronique et formelle. Cahiers Chronos, 16: 121-151.

Dahl, O. (1997), The relation between past time reference and counterfactuality: A new look. In: A. Athanasiadou et R. Dirven (eds), On conditionals again, Amsterdam / Philadelphia: John Benjamins, pp. 97-112.

Dendale, P. (2001). Les problèmes linguistiques du conditionnel en français. In: P. Dendale et L. Tasmowski (eds), Le conditionnel en français. Paris: Klincksieck, pp. 7-18.

Goldberg, A. E., 2006, Constructions at Work. The Nature of Generalization in Langage. Oxford: Oxford University Press.

Gosselin, L. (1999). Les valeurs de l'imparfait et du conditionnel dans les systèmes hypothétiques, Cahiers Chronos, 4: 29-51.

Gosselin, L. (2005). Temporalité et modalité. Bruxelles: Duculot.

Gosselin, L. (2009). Les modalités en français. Amsterdam / New York: Rodopi.

Haillet, P. P. (2002). Le conditionnel en français : une approche polyphonique. Gap: Ophrys.

Harris, M. (1978). The Evolution of French Syntax: A Comparative Approach. Londres: Longman.

Heine, B. (2002). On the Role of Contexts in Grammaticalization. In: I. Wischer et G. Diewald (eds), New Reflections on Grammaticalization. Amsterdam: John Benjamins, pp. 83-101.

Kronning, H., (2009). Polyphonie, constructions conditionnelles et discours rapporté, Langue française, 164: 97-112.

Kronning, H. (2014). Pour une linguistique contrastive variationnelle : le conditionnel épistémique d'emprunt en français, en italien et en espagnol. In: H. P. Helland et Ch. Melklenborg Salvesen (eds), Affaire(s) de grammaire. Mélanges offerts à Marianne Hobak Haff à l'occasion de ses soixante-cinq ans. Oslo: Novus Forlag, pp. 67-90.

Marchello-Nizia, C. (2006). Grammaticalisation et changement linguistique. Bruxelles: De Boeck.

Mourelatos A., 1978, Events, Processes and States, Linguistics and Philosophy, 2: 415-434. 
Patard, A. (2007). L'un et le multiple. L'imparfait de l'indicatif en français : valeur en langue et usages en discours. Thèse de doctorat, Université Paul-Valéry - Montpellier 3.

Patard, A. et De Mulder, W. (2012). Le développement du conditionnel en français. Faits de langue, 40: 29-36.

Patard, A. et De Mulder, W. (2014). Aux origines des emplois modaux de l'imparfait: le cas des emplois contrefactuel et hypothétique. Langages, 193: 33-47.

Vetters, C. (2010). Développement et évolution des temps du passé en français : passé simple, passé composé et venir de +infinitif. Cahiers Chronos, 21: 277-298.

Wagner, R.-L. (1939). Les Phrases hypothétiques commençant par « si » dans la langue française, des origines à la fin du XVIe siècle. Paris: Droz.

Yvon, H. (1952). Faut-il distinguer deux conditionnels dans le verbe français ?, Le Français Moderne, 20: 249-265.

Yvon, H. (1958). Supposition, subjonctif et conditionnel. Le français moderne, 26.3: 161-183. 